\title{
13. GEOCHEMISTRY OF SEDIMENTS AND AUTHIGENIC MINERALS IN AND AROUND THE DÉCOLLEMENT ZONE OF THE NORTHERN BARBADOS RIDGE ACCRETIONARY PRISM ${ }^{1}$
}

\author{
T. Ito, ${ }^{2}$ K. Yamaguchi, ${ }^{3}$ K. Komuro, ${ }^{3}$ Y. Ogawa ${ }^{3}$
}

\begin{abstract}
Chemical analyses for major and trace constituents of authigenic minerals and sediments collected in the northern Barbados Ridge during Ocean Drilling Program Leg 156 have been carried out. In addition to Mn enrichments previously reported, $\mathrm{Cu}$ enrichments were identified in this study.

Two kinds of Mn enrichments of rhodochrosite and amorphous Mn oxide occur at Site 948. Both of them have rare-earth element (REE) abundances similar to the North American Shale Composites and typical sediments recovered during Leg 156. $\mathrm{Cu}$ enrichments occur just above the décollement zone at Site 948 , and also just above an inferred thrust based on the drastic change in interstitial-water chemistry at Site 949 . The $\mathrm{Cu}$-enriched parts are characterized by $\mathrm{K}, \mathrm{Ba}, \mathrm{Cr}$, and light REE depletions.
\end{abstract}

\section{INTRODUCTION}

The northern Barbados Ridge accretionary complex (Figs. 1,2) is an ideal area to study the origin and migration processes of water and accompanying elements in accretionary complexes, because drilling has penetrated the décollement zone (Legs 78A, 110, and 156). In recent years, much attention has been paid to geochemical studies of fluids and authigenic minerals in accretionary complexes to achieve this scientific goal (e.g., Gieskes et al., 1990; Vrolijk and Sheppard, 1991; Kastner et al., 1991). Although trace-element compositions of authigenic minerals and sediments are also important in the understanding of geochemistry during accretionary processes, only the major-element chemistry of bulk sediments was presented for the Barbados accretionary complex by Wang et al. (1990). Here we report the trace-element chemical compositions, including rare-earth element (REE) abundances, in addition to major constituents for authigenic minerals and bulk sediments.

\section{METHODS}

Sediment samples composed of several color fractions were separated macroscopically, without washing. Each sample was powdered using an agate mortar after drying at $60^{\circ} \mathrm{C}$ overnight, and was completely dissolved by adding $\mathrm{HNO}_{3}(0.5 \mathrm{ml}), \mathrm{HClO}_{4}(0.2 \mathrm{ml})$ and $\mathrm{HF}(1 \mathrm{ml})$ for all samples except amorphous Mn-enriched samples, and $\mathrm{HCl}(1 \mathrm{ml}), \mathrm{HNO}_{3}(0.5 \mathrm{ml}), \mathrm{HClO}_{4}(0.2 \mathrm{ml})$, and $\mathrm{HF}(0.2 \mathrm{ml})$ for amorphous Mn-enriched samples to closed teflon vials for $10 \mathrm{hr}$ at $110^{\circ} \mathrm{C}$, after weighing samples accurately (nearly $80 \mathrm{mg}$ ). The sample solutions were dried in an open system and were made up in a final solution of $5 \% \mathrm{HNO}_{3}$. All element compositions except REEs were analyzed using inductively coupled plasma-atomic emission spectroscopy (ICP-AES, Jarrell Ash ICAP-757V). REEs were analyzed using inductively coupled plasma-mass spectrometry (ICPMS, Yokogawa Hewlett Packard PMS2000) at the Chemical Analy-

${ }^{1}$ Shipley, T.H., Ogawa, Y., Blum, P., and Bahr, J.M. (Eds.), 1997. Proc. ODP, Sci. Results, 156: College Station, TX (Ocean Drilling Program).

${ }^{2}$ Faculty of Education, Ibaraki University, Mito, Ibaraki, 310, Japan. tito@mito.ipc.ibaraki.ac.jp

${ }^{3}$ Institute of Geoscience, University of Tsukuba, Tsukuba, Ibaraki, 305, Japan. sis Center, the University of Tsukuba. Calibrations were performed using solution standards and standard rock samples from the Geological Survey of Japan (JGb1, JG1a, JG2, JB1a, JB2, and JSd3) for REEs and the other elements analyses.

Data on the chemical composition of sediments of Sites 948 and 949 are given in Tables 1 and 2. REE contents are presented in Table 3.

\section{CHEMICAL COMPOSITION}

Lithostratigraphic summaries with authigenic mineral occurrences for Sites 948 and 949 are presented in Figures 3 and 4.

\section{Site 948}

The chemical compositions of authigenic minerals and bulk sediments of Sites 948 are presented in Figure 5.

The major constituent $\mathrm{Al}, \mathrm{Fe}, \mathrm{Mg}$, and $\mathrm{Na}$ have nearly constant values throughout the core. Ca content varies depending on the contribution of fossiliferous carbonates. K content decreases near the upper boundary of the décollement zone, but decreases at $566.9 \mathrm{~m}$ below seafloor (mbsf) because of dilution by high carbonate contents.

Contents of four elements $(\mathrm{Mn}, \mathrm{Cu}, \mathrm{Cr}$, and $\mathrm{Ba}$ ) fluctuate greatly at Site 948.

Mn enrichments ( 3.5-13 wt\%) at Site 948 are characterized by two types. One is white in color and is composed of rhodochrosite, identified using X-ray powder diffraction. This first type of Mn enrichment occurs at 423.2 and $472.7 \mathrm{mbsf}$, which corresponds to a horizon above the décollement zone. The second type of Mn enrichment is black (Fig. 6). This type of manganese enrichment is amorphous, using X-ray powder diffraction, and occurs at 500 and $509.3 \mathrm{mbsf}$ within the décollement zone. The second types have high Ba contents (1400-2200 ppm). Both types of Mn-enriched rhodochrosite and amorphous Mn oxides also have REE patterns similar to the North American Shale Composites (NASC) and typical sediments from Leg 156, regardless of mineralogy (Fig. 7).

$\mathrm{Cu}$ enrichment $(\sim 1.3 \mathrm{wt} \%)$ was first identified in the accretionary complexes. At Site 948, Cu enrichment occurs at 494.9 mbsf, which corresponds to just above the décollement zone. Chemically, the depletion of $\mathrm{K}, \mathrm{Ba}$, and $\mathrm{Cr}$ are characteristic of the $\mathrm{Cu}$-enriched sediments. In addition, these sections are also depleted in REEs, especially in light REEs (Fig. 8). 

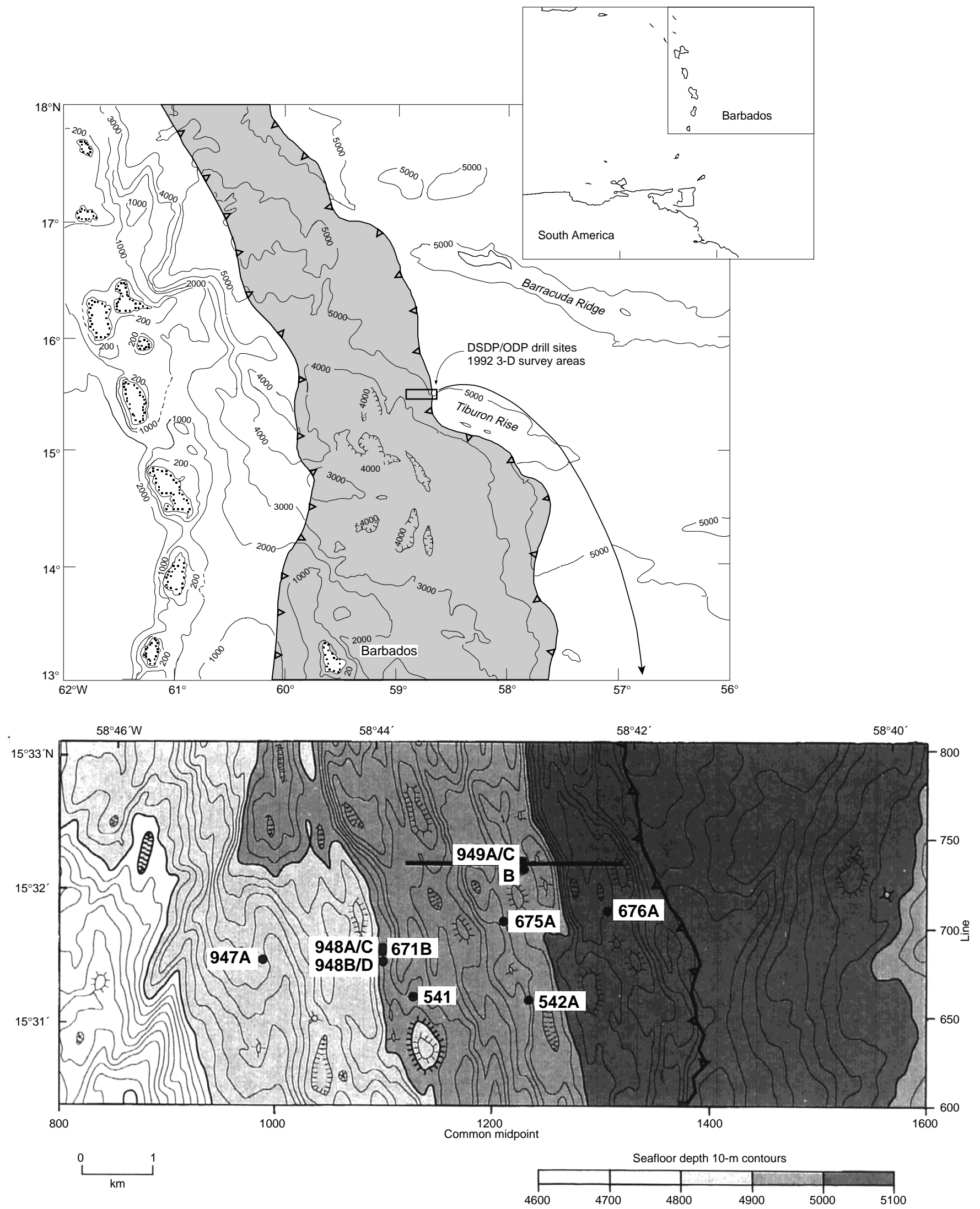

Figure 1. Location map showing the area around Sites 948 and 949. The frontal thrust (barbed line) and Deep Sea Drilling Project (DSDP) and other drill sites are shown. Modified from Shipboard Scientific Party (1995a, 1995c). 


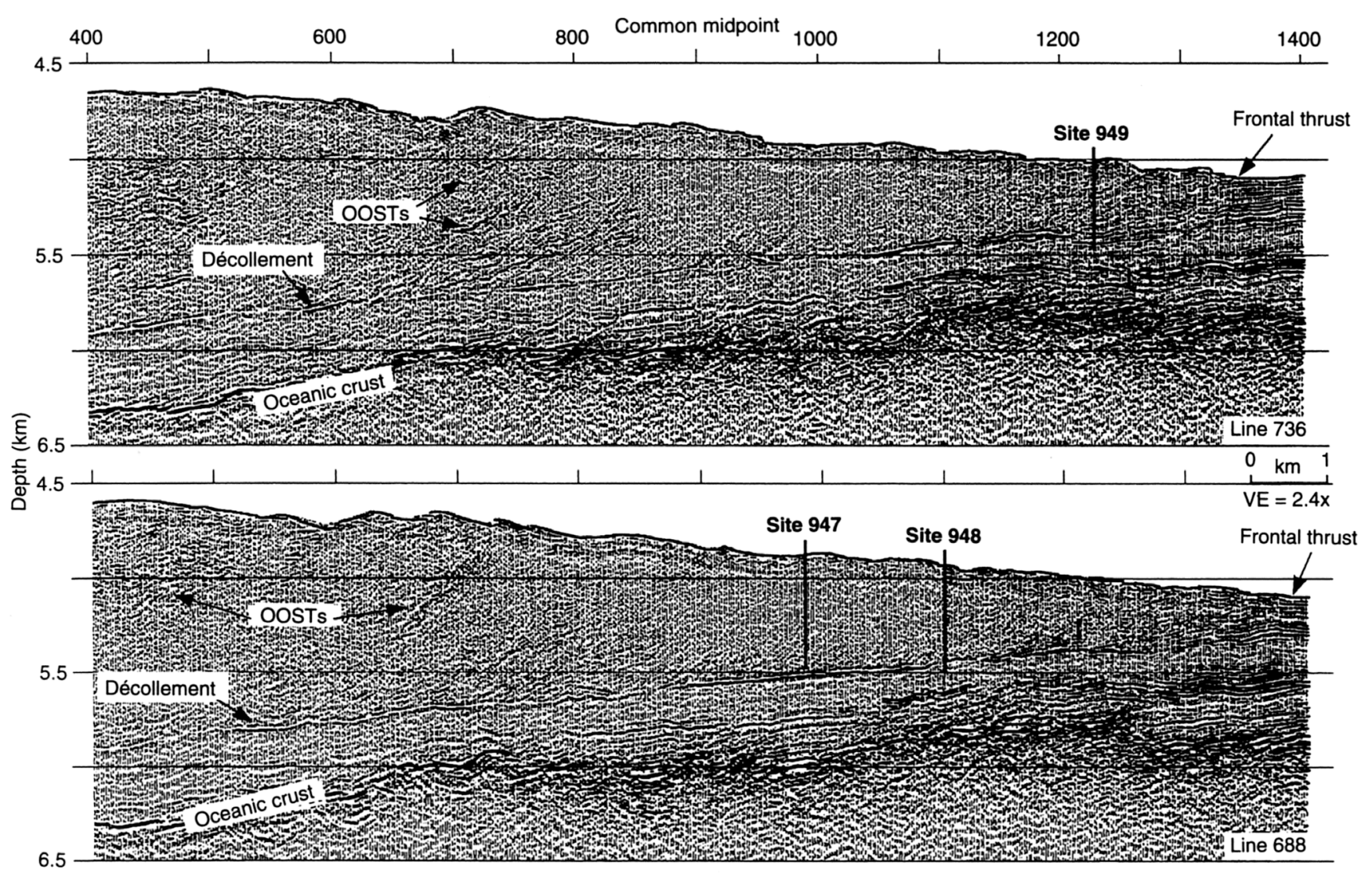

Figure 2. Depth section of three-dimensional seismic images around Sites 948 and 949. This figure is adopted from Moore et al. (1995).

The vertical profile of $\mathrm{Cr}$ content is similar to the K-content pattern at Site 948 . From similar values at 590 to $500 \mathrm{mbsf}$, Cr content decreases abruptly from 90 to $10 \mathrm{ppm}$ at the upper boundary of the décollement zone, and increases monotonically toward the upper layer. Several Cr-depleted parts are also poor in REEs, especially in light REEs, regardless of $\mathrm{Cu}$ content (Fig. 8).

\section{Site 949}

The chemical composition of bulk sediments and authigenic mineral of Sites 949 is presented in Figure 9.

It is hard to understand totally the trend of chemical composition at Site 949 because of poor core recovery. The ranges of fluctuation are similar to those at Site 948 for most of the elements.

Although no abnormal $\mathrm{Mn}$ enrichment occurs at this site, $\mathrm{Cu}$ enrichment ( $3.3 \mathrm{wt} \%)$ was present at 284.4 mbsf (Fig. 10), which corresponds to just above the inferred thrust, where the chemical composition of interstitial water changed drastically (Shipboard Scientific Party, 1995c). Similar to the case at Site 948, the Cu-enriched part was characteristically depleted in $\mathrm{K}, \mathrm{Ba}, \mathrm{Cr}$, and light REEs.

\section{SUMMARY}

Studies of bulk chemical composition have revealed geochemical features of sediments recovered from Leg 156 sites. Several important characteristics have been identified.

1. Both types of Mn enrichments, rhodochrosite and amorphous Mn oxide, are identical to the NASC and typical sediments from Leg 156 in terms of REE abundance. Only black amorphous Mn oxide is accompanied by a Ba enrichment.

2. Cu-enriched parts $(\sim 1.3-3.3 \mathrm{wt} \%)$ were identified from around the décollement zone or thrust, indicated by drastic changes in interstitial-water chemistry. They are characterized chemically by $\mathrm{K}, \mathrm{Ba}, \mathrm{Cr}$, and light REE depletions.

\section{ACKNOWLEDGMENTS}

The authors would like to thank Ms. Y. Takeuchi of the Chemical Analysis Center, the University of Tsukuba, for supporting the chemical analyses.

\section{REFERENCES}

Gieskes, J.M., Vrolijk, P., and Blanc, G., 1990. Hydrogeochemistry of the northern Barbados accretionary complex transect: Ocean Drilling Program Leg 110. J. Geophys. Res., 95:8809-8818.

Gromet, L.P., Dymek, R.F., Haskin, L.A., and Korotev, R.L., 1984. The "North American Shale Composite": its compilation, major and trace elements characteristics. Geochim. Cosmochim. Acta, 48:2469-2482.

Haskin, L.A., Haskin, M.A., Frey, F.A., and Wildman, T.R., 1968. Relative and absolute terrestrial abundances of the rare earths. In Ahrens, L.H. (Ed.), Origin and Distribution of the Elements: New York (Pergamon), 889-912.

Kastner, M, Elderfield, H. Martin, J. B., 1991. Fluids in convergent margins: what do we know about their composition, origin, role in diagenesis and importance for oceanic chemical fluxes? Phil. Trans. R. Soc. Lond. A, 335: 243-259.

Moore, G.F., Zhao, Z., Shipley, T.H., Bangs, N., and Moore, J.C., 1995. Structural setting of the Leg 156 area, northern Barbados Ridge accre- 
tionary prism. In Shipley, T.H., Ogawa, Y., Blum, P., et al., Proc. ODP, Init. Repts., 156: College Station, TX (Ocean Drilling Program), 13-27.

Shipboard Scientific Party, 1995a. Introduction. In Shipley, T.H., Ogawa, Y., Blum, P., et al., Proc. ODP, Init. Repts., 156: College Station, TX (Ocean Drilling Program), 3-11.

, 1995b. Site 948. In Shipley, T.H., Ogawa, Y., Blum, P., et al., Proc. ODP, Init. Repts., 156: College Station, TX (Ocean Drilling Program), 87-192.

1995c. Site 949. In Shipley, T.H., Ogawa, Y., Blum, P., et al., Proc. ODP, Init. Repts., 156: College Station, TX (Ocean Drilling Program), 193-257.
Vrolijk, P., and Sheppard, S.M.E., 1991. Syntectonic carbonate veins from the Barbados accretionary prism (ODP Leg 110): record of palaeohydrology. Sedimentology, 38:671-690.

Wang, Y.C., Gieskes, J.M., and Musoke, L., 1990. Bulk chemical analysis of sediments-Hole 671B. In Moore, J.C., Mascle, A. et al., Proc. ODP, Sci. Results, 110: College Station, TX (Ocean Drilling Program), 179-188.

Date of initial receipt: 5 February 1996

Date of acceptance: 16 July 1996

Ms 156SR-022 
Table 1. Chemical composition of bulk sediments and authigenic minerals, Site 948.

\begin{tabular}{|c|c|c|c|c|c|c|c|c|c|c|c|c|c|c|c|c|c|c|}
\hline $\begin{array}{l}\text { Core, section, } \\
\text { interval }(\mathrm{cm})\end{array}$ & $\begin{array}{r}\text { Depth } \\
\text { (mbsf) }\end{array}$ & $\begin{array}{l}\text { Working } \\
\text { no. }\end{array}$ & $\begin{array}{l}\mathrm{Al} \\
(\%)\end{array}$ & $\begin{array}{c}\mathrm{Ba} \\
(\mathrm{ppm})\end{array}$ & $\begin{array}{l}\mathrm{Ca} \\
(\%)\end{array}$ & $\underset{(\mathrm{ppm})}{\mathrm{Cr}}$ & $\begin{array}{c}\mathrm{Cu} \\
(\mathrm{ppm})\end{array}$ & $\begin{array}{l}\mathrm{Fe} \\
(\%)\end{array}$ & $\begin{array}{c}\mathrm{K} \\
(\%)\end{array}$ & $\begin{array}{c}\mathrm{Li} \\
(\mathrm{ppm})\end{array}$ & $\begin{array}{l}\mathrm{Mg} \\
(\%)\end{array}$ & $\begin{array}{l}\mathrm{Mn} \\
(\%)\end{array}$ & $\begin{array}{l}\mathrm{Na} \\
(\%)\end{array}$ & $\begin{array}{c}\mathrm{P} \\
(\mathrm{ppm})\end{array}$ & $\begin{array}{c}\mathrm{Sr} \\
(\mathrm{ppm})\end{array}$ & $\begin{array}{c}\mathrm{Ti} \\
(\mathrm{ppm})\end{array}$ & $\begin{array}{c}\mathrm{V} \\
(\mathrm{ppm})\end{array}$ & $\begin{array}{c}\mathrm{Zn} \\
(\mathrm{ppm})\end{array}$ \\
\hline $\begin{array}{l}156-948 \mathrm{~B}- \\
1 \mathrm{H}-3,54-56\end{array}$ & 3.5 & 1 & 6.76 & 278 & 1.31 & 87 & 80 & 3.79 & 1.53 & 53 & 1.27 & 0.123 & 1.66 & 504 & 596 & 3,202 & 125 & 90 \\
\hline \multicolumn{19}{|l|}{$156-948 \mathrm{C}-$} \\
\hline $\begin{array}{l}1 \mathrm{H}-2,89-91 \\
1 \mathrm{H}-6,99-101\end{array}$ & 8.5 & 3 & $\begin{array}{r}9.04 \\
10.12\end{array}$ & 393 & $\begin{array}{l}1.00 \\
1.76\end{array}$ & 121 & 71 & $\begin{array}{l}. .44 \\
5.82\end{array}$ & $\begin{array}{l}1.93 \\
2.03\end{array}$ & 78 & $\begin{array}{l}1.12 \\
1.69\end{array}$ & $\begin{array}{l}0.509 \\
0.108\end{array}$ & $\begin{array}{l}1.01 \\
1.76\end{array}$ & $\begin{array}{l}000 \\
590\end{array}$ & $\begin{array}{l}184 \\
182\end{array}$ & $\begin{array}{r}4,180 \\
4,780\end{array}$ & 177 & 138 \\
\hline $2 \mathrm{X}-2,85-87$ & 423.2 & 4 & 8.74 & 253 & 1.25 & 123 & 27 & 5.30 & 1.85 & 59 & 1.71 & 6.000 & 0.91 & 720 & 102 & 3,733 & 135 & 120 \\
\hline $3 X-3,13-15$ & 436.6 & 5 & 10.31 & 278 & 0.80 & 88 & 62 & 5.56 & 1.97 & 73 & 1.84 & 0.157 & 1.39 & 584 & 118 & 5,181 & 183 & 138 \\
\hline $4 X-3,102-106$ & 444.1 & 6 & 9.29 & 225 & 4.83 & 81 & 67 & 4.70 & 1.65 & 74 & 1.81 & 0.137 & 1.24 & 396 & 257 & 4,237 & 180 & 114 \\
\hline $4 X-4,63-66$ & 445.2 & 7 & 7.63 & 245 & 11.26 & 89 & 36 & 4.26 & 1.62 & 63 & 1.27 & 0.534 & 0.78 & 519 & 525 & 3,065 & 111 & 95 \\
\hline $5 X-4,137-139$ & 455.7 & 8 & 9.64 & 213 & 1.57 & 77 & 53 & 5.04 & 1.64 & 84 & 1.79 & 0.155 & 1.26 & 350 & 149 & 4,650 & 129 & 100 \\
\hline $6 \mathrm{X}-2,61-63$ & 461.5 & 10 & 10.69 & 241 & 0.54 & 83 & 80 & 5.94 & 1.79 & 106 & 1.69 & 0.156 & 1.10 & 446 & 103 & 4,478 & 120 & 119 \\
\hline $6 \mathrm{X}-3,127-129$ & 463.7 & 11 & 9.52 & 179 & 0.86 & 53 & 38 & 5.29 & 1.38 & 96 & 2.11 & 0.293 & 1.49 & 427 & 100 & 4,413 & 140 & 109 \\
\hline $7 \mathrm{X}-3,71-73$ & 472.7 & 12 & 6.69 & 193 & 2.53 & 76 & 203 & 3.25 & 1.14 & 67 & 1.89 & 13.150 & 0.94 & 469 & 85 & 3,222 & 109 & 92 \\
\hline $7 \mathrm{X}-3,123-126$ & 473.2 & 13 & 9.77 & 229 & 0.72 & 59 & 48 & 5.63 & 1.45 & 88 & 2.00 & 0.156 & 1.42 & 435 & 106 & 4,947 & 133 & 104 \\
\hline $8 X-5,122-124$ & 485.9 & 14 & 9.24 & 288 & 1.33 & 49 & 76 & 6.39 & 1.18 & 84 & 2.05 & 0.120 & 1.67 & 433 & 127 & 5,168 & 108 & 117 \\
\hline $9 X-4,6-9$ & 493.0 & 15 & 9.56 & 37 & 2.29 & 10 & 151 & 4.56 & 0.5 & 35 & 1.90 & 0.116 & 2.01 & 791 & 407 & 5,272 & 55 & 76 \\
\hline $9 \mathrm{X}-4,27-30$ & 493.2 & 16 & 8.68 & 289 & 1.23 & 47 & 52 & 5.82 & 1.06 & 74 & 2.17 & 0.353 & 1.63 & 479 & 120 & 4,996 & 147 & 110 \\
\hline $9 \mathrm{X}-5,53-55$ & 494.9 & 17 & $\begin{array}{l}8.00 \\
8.07\end{array}$ & 43 & 2.21 & 12 & 13,000 & $\begin{array}{l}6.02 \\
6.45\end{array}$ & 1.23 & 41 & 1.61 & 0.098 & 2.04 & 141 & 141 & 6,926 & 385 & 177 \\
\hline $10 X-1,74-77$ & 498.8 & 18 & 8.65 & 57 & 2.77 & 42 & 61 & 6.77 & 0.66 & 36 & 1.44 & 0.078 & 2.20 & 233 & 201 & 6,352 & 301 & 115 \\
\hline \multirow{2}{*}{$10 \mathrm{X}-2,44-46$} & 500.0 & 19ocher & 9.34 & 217 & 1.18 & 71 & 99 & 4.87 & 1.30 & 81 & 1.68 & 0.193 & 1.64 & 629 & 152 & 3,907 & 111 & 123 \\
\hline & 500.0 & 19black & 8.31 & 1,454 & 1.16 & 80 & 127 & 4.70 & 1.19 & 73 & 1.62 & 4.763 & 1.62 & 570 & 183 & 3,726 & 303 & 121 \\
\hline $10 \mathrm{X}-2,57-59$ & 500.2 & 20 & 9.23 & 259 & 1.10 & 79 & 92 & 5.26 & 1.24 & 75 & 1.59 & 0.374 & 1.44 & 553 & 144 & 4,295 & 136 & 126 \\
\hline $10 \mathrm{X}-3,31-33$ & 501.4 & 21 & 9.39 & 272 & 0.99 & 90 & 95 & 4.95 & 1.23 & 78 & 1.57 & 0.337 & 1.41 & 490 & 127 & 4,040 & 137 & 128 \\
\hline \multirow{3}{*}{$\begin{array}{l}10 X-3,110-150 \\
11 X-2,13-15\end{array}$} & 502.4 & 22 & 8.85 & 467 & 0.82 & 88 & 91 & 4.70 & 1.16 & 78 & 1.59 & 0.729 & 1.29 & 415 & 125 & 3,735 & 150 & 124 \\
\hline & 509.3 & 24ocher & 9.65 & 423 & 0.50 & 100 & 238 & 4.90 & 1.33 & 67 & 1.13 & 0.149 & 1.07 & 408 & 116 & 3,814 & 131 & 122 \\
\hline & 509.3 & 24black & 9.03 & 2,128 & 0.53 & 89 & 430 & 4.75 & 1.25 & 63 & 1.17 & 3.677 & 1.06 & 467 & 170 & 3,748 & 241 & 128 \\
\hline $11 X-5,25-27$ & 514.0 & 25 & 8.48 & 288 & 0.32 & 76 & 37 & 5.01 & 1.42 & 42 & 0.96 & 0.038 & 1.18 & 209 & 108 & 4,008 & 150 & 96 \\
\hline $12 X-5,76-80$ & 524.2 & 26 & 8.80 & 429 & 0.48 & 97 & 42 & 3.95 & 1.7 & 75 & 0.94 & 0.078 & 1.09 & 34 & 11 & 4,343 & 167 & 187 \\
\hline \multirow[t]{2}{*}{12} & 524.9 & 27 greenish & 10.03 & 333 & 0.34 & 98 & 46 & 5.03 & 2.18 & 72 & 1.26 & 0.025 & 0.95 & 224 & 107 & 3,809 & 163 & 106 \\
\hline & 524.9 & 27 gray & 10.55 & 320 & 0.30 & 137 & 47 & 4.02 & 2.32 & 81 & 1.05 & 0.023 & 1.01 & 177 & 117 & 3,425 & 163 & 136 \\
\hline \multirow[t]{2}{*}{$13 \mathrm{X}-\mathrm{CC}, 7-9$} & 536.4 & 28greenish & 11.03 & 399 & 0.31 & 101 & 39 & 5.01 & 2.21 & $\begin{array}{l}01 \\
100\end{array}$ & $\begin{array}{l}1.05 \\
1.09\end{array}$ & 0.018 & 0.86 & 32 & 109 & 4,177 & 188 & 133 \\
\hline & 536.4 & 28 gray & 11.75 & 324 & 0.29 & 105 & 34 & 4.12 & 2.45 & 104 & 0.98 & 0.020 & 0.90 & 202 & 132 & 4,300 & 210 & 154 \\
\hline $14 \mathrm{X}-2,17-19$ & 537.8 & 29 & 8.86 & 312 & 0.32 & 394 & 29 & 13.16 & 2.20 & 89 & 0.83 & 0.021 & 0.77 & 245 & 943 & 2,541 & 70 & 128 \\
\hline $14 X-5,48-50$ & 542.6 & 30 & 11.11 & 368 & 0.32 & 106 & 28 & 5.54 & 2.26 & 102 & 1.24 & 0.019 & 0.86 & 259 & 109 & 4,183 & 190 & 120 \\
\hline $15 X-2,87-89$ & 547.8 & 31 & 11.39 & 546 & 0.39 & 121 & 120 & 4.99 & 1.44 & 132 & 1.25 & 0.022 & 0.88 & 419 & 954 & 933 & 175 & 277 \\
\hline $15 X-7,25-27$ & 554.7 & 32 & 9.57 & 335 & 0.36 & 97 & 38 & 6.96 & 2.36 & 72 & 1.23 & 0.037 & 0.88 & 292 & 114 & 4,082 & 162 & 108 \\
\hline $17 \mathrm{X}-2,120-150$ & 566.9 & 33 & 1.89 & 1,180 & 24.60 & 38 & 18 & 2.78 & 0.31 & 37 & 0.65 & 1.042 & 0.46 & 357 & 1,186 & 3,506 & 50 & 33 \\
\hline $17 \mathrm{X}-4,81-84$ & 569.4 & 34 & 10.41 & 370 & 2.61 & 118 & 129 & 4.55 & 174 & 120 & 1.12 & 0029 & 0.90 & 536 & 218 & 4355 & 168 & 137 \\
\hline $\begin{array}{l}11 \mathrm{X}-4,68-74 \\
18 \mathrm{X}-2,68-70\end{array}$ & $\begin{array}{l}309.4 \\
575.5\end{array}$ & $\begin{array}{l}34 \\
35\end{array}$ & $\begin{array}{r}10.41 \\
9.68\end{array}$ & 385 & $\begin{array}{l}2.01 \\
0.37\end{array}$ & $\begin{array}{r}118 \\
99\end{array}$ & 46 & 3.88 & 2.11 & $\begin{array}{r}120 \\
86\end{array}$ & 0.99 & $\begin{array}{l}.029 \\
0.019\end{array}$ & 0.91 & $\begin{array}{l}530 \\
288\end{array}$ & 120 & $\begin{array}{l}4,3,5 \\
5,197\end{array}$ & $\begin{array}{l}108 \\
192\end{array}$ & 177 \\
\hline $19 X-6,72-75$ & 591.0 & 36 & 8.18 & 53 & 10.24 & 11 & 57 & 4.3 & 1.4 & 81 & 0.96 & 0.131 & 0.75 & 3 & 656 & 3,555 & 133 & 105 \\
\hline $19 X-7,33-36$ & 592.1 & 37 & 10.34 & 338 & 0.93 & 100 & 40 & 5.39 & 2.26 & 86 & 1.13 & 0.092 & 0.79 & 288 & 161 & 4,943 & 175 & 390 \\
\hline
\end{tabular}




\begin{tabular}{|c|c|c|c|c|c|c|c|c|c|c|c|c|c|c|c|c|c|c|}
\hline $\begin{array}{l}\text { Core, section, } \\
\text { interval }(\mathrm{cm})\end{array}$ & $\begin{array}{l}\text { Depth } \\
\text { (mbsf) }\end{array}$ & $\begin{array}{l}\text { Working } \\
\text { no. }\end{array}$ & $\begin{array}{c}\mathrm{Al} \\
(\%)\end{array}$ & $\begin{array}{c}\mathrm{Ba} \\
(\mathrm{ppm})\end{array}$ & $\begin{array}{l}\mathrm{Ca} \\
(\%)\end{array}$ & $\begin{array}{c}\mathrm{Cr} \\
(\mathrm{ppm})\end{array}$ & $\begin{array}{c}\mathrm{Cu} \\
(\mathrm{ppm})\end{array}$ & $\begin{array}{l}\mathrm{Fe} \\
(\%)\end{array}$ & $\begin{array}{c}\mathrm{K} \\
(\%)\end{array}$ & $\begin{array}{c}\mathrm{Li} \\
(\mathrm{ppm})\end{array}$ & $\begin{array}{l}\mathrm{Mg} \\
(\%)\end{array}$ & $\begin{array}{l}\mathrm{Mn} \\
(\%)\end{array}$ & $\begin{array}{l}\mathrm{Na} \\
(\%)\end{array}$ & $\underset{(\mathrm{ppm})}{\mathrm{P}}$ & $\begin{array}{c}\mathrm{Sr} \\
(\mathrm{ppm})\end{array}$ & $\begin{array}{c}\mathrm{Ti} \\
(\mathrm{ppm})\end{array}$ & $\begin{array}{c}\mathrm{V} \\
(\mathrm{ppm})\end{array}$ & $\begin{array}{c}\mathrm{Zn} \\
(\mathrm{ppm})\end{array}$ \\
\hline $\begin{array}{l}\text { 156-949A- } \\
1 \mathrm{H}-2,61-64\end{array}$ & 2.1 & 38 & 8.21 & 407 & 3.75 & 99 & 98 & 4.91 & 2.14 & 67 & 1.63 & 0.320 & 2.11 & 454 & 236 & 3,962 & 151 & 104 \\
\hline $\begin{array}{l}156-949 \mathrm{~B}- \\
1 \mathrm{X}-29-11\end{array}$ & & & & & & & & & & & & & & & & & & \\
\hline $\begin{array}{l}1 \mathrm{X}-2,9-11 \\
2 \mathrm{X}-6.58-60\end{array}$ & $\begin{array}{l}243.1 \\
2619\end{array}$ & $\begin{array}{l}39 \\
40\end{array}$ & $\begin{array}{r}9.37 \\
1027\end{array}$ & $\begin{array}{l}225 \\
246\end{array}$ & $\begin{array}{l}0.95 \\
0.52\end{array}$ & $\begin{array}{l}87 \\
10\end{array}$ & $\begin{array}{l}45 \\
74\end{array}$ & $\begin{array}{r}5.40 \\
66.07\end{array}$ & $\begin{array}{l}1.71 \\
1.79\end{array}$ & $\begin{array}{l}79 \\
95\end{array}$ & $\begin{array}{l}1.80 \\
159\end{array}$ & 0.160 & $\begin{array}{l}1.35 \\
1.18\end{array}$ & $\begin{array}{l}369 \\
572\end{array}$ & 117 & 4,281 & 139 & 325 \\
\hline $\begin{array}{l}2 \mathrm{X}-6,58-60 \\
3 \mathrm{X}-3,135-137\end{array}$ & $\begin{array}{l}201.9 \\
267.9\end{array}$ & 41 & 10.21 & $\begin{array}{l}240 \\
243\end{array}$ & 0.64 & $\begin{array}{l}10 \\
105\end{array}$ & $\begin{array}{l}14 \\
55\end{array}$ & $\begin{array}{r}0.07 \\
5.61\end{array}$ & $\begin{array}{l}1.19 \\
1.81\end{array}$ & $\begin{array}{l}93 \\
93\end{array}$ & $\begin{array}{l}1.59 \\
1.74\end{array}$ & $\begin{array}{l}0.349 \\
0.189\end{array}$ & $\begin{array}{l}1.18 \\
1.23\end{array}$ & $\begin{array}{l}572 \\
785\end{array}$ & $\begin{array}{l}101 \\
105\end{array}$ & $\begin{array}{l}4,240 \\
4223\end{array}$ & & $\begin{array}{l}133 \\
117\end{array}$ \\
\hline $\begin{array}{l}3 \mathrm{X}-3,135-137 \\
4 \mathrm{X}-\mathrm{CC}, 19-21\end{array}$ & 275.0 & 42 & 9.47 & 157 & 0.84 & 44 & 345 & 3.45 & 1.46 & 90 & 2.31 & $\begin{array}{l}0.189 \\
0.180\end{array}$ & $\begin{array}{l}1.23 \\
1.89\end{array}$ & $\begin{array}{l}785 \\
260\end{array}$ & $\begin{array}{l}105 \\
142\end{array}$ & $\begin{array}{l}4,223 \\
5,215\end{array}$ & $\begin{array}{l}143 \\
100\end{array}$ & 122 \\
\hline $5 X-2,8-10$ & 284.4 & 43greenish & 7.97 & 12 & 1.40 & 11 & 33,400 & 2.31 & 0.53 & 64 & 2.28 & 0.128 & 1.73 & 431 & 119 & 3,718 & 129 & 189 \\
\hline & 284.4 & 43matrics & 8.26 & 192 & 0.84 & 25 & 97 & 3.90 & 0.86 & 77 & 2.41 & 0.131 & 1.81 & 240 & 110 & 4,530 & 194 & 102 \\
\hline $5 X-5,3-5$ & 288.8 & 44 & 8.81 & 426 & 0.83 & 78 & 69 & 4.85 & 1.68 & 80 & 1.88 & 0.210 & 1.71 & 419 & 149 & 4,124 & 147 & 121 \\
\hline $5 X-7,40-42$ & 292.2 & 45 & 8.44 & 311 & 0.99 & 55 & 88 & 5.32 & 1.13 & 66 & 2.05 & 0.191 & 1.6 & 50 & 125 & 4,190 & 118 & 122 \\
\hline $7 X-2,60-62$ & 304.2 & 46 & 9.65 & 668 & 2.84 & 7 & 43 & 1.66 & 0.86 & 49 & 1.95 & 0.103 & 2.2 & 19 & 24 & 2,697 & 108 & 93 \\
\hline $13 \mathrm{X}-2,36-39$ & 352.1 & 47 & 9.68 & 196 & 1.15 & 76 & 51 & 5.11 & 1.69 & 87 & 1.82 & 0.195 & 1.46 & 449 & 12 & 4,400 & 138 & 113 \\
\hline $13 \mathrm{X}-2,92-96$ & 352.6 & 48 & 10.88 & 232 & 0.50 & 110 & 58 & 5.49 & 2.48 & 110 & 1.57 & 0.085 & 1.33 & 471 & 147 & 4,591 & 150 & 137 \\
\hline $14 \mathrm{X}-3,72-77$ & 358.6 & 49 & 9.44 & 337 & 0.70 & 34 & 241 & 4.87 & 1.36 & 84 & 1.89 & 0.233 & 1.89 & 182 & 109 & 2,848 & 82 & 93 \\
\hline $14 \mathrm{X}-6,67-69$ & 363.1 & 50 & 10.35 & 221 & 0.48 & 82 & 31 & 5.89 & 1.83 & 91 & 1.60 & 0.081 & 1.23 & 469 & 108 & 4,499 & 123 & 108 \\
\hline $15 \mathrm{X}-2,48-50$ & 361.9 & 51 & 9.73 & 183 & 0.58 & 72 & 49 & 5.15 & 1.58 & 95 & 1.96 & 0.159 & 1.48 & 391 & 106 & 4,242 & 142 & 119 \\
\hline $15 \mathrm{X}-4,21-23$ & 364.6 & 52 & 9.37 & 192 & 0.61 & 64 & 84 & 5.12 & 1.45 & 89 & 2.08 & 0.197 & 1.43 & 390 & 108 & 4,155 & 116 & 113 \\
\hline $19 \mathrm{X}-3,20-22$ & 401.5 & 53 & 9.31 & 216 & 0.60 & 92 & 120 & 4.83 & 1.13 & 84 & 1.44 & 0.199 & 1.33 & 371 & 125 & 3,898 & 118 & 138 \\
\hline $19 X-3,51-53$ & 401.8 & 54ocher & 9.45 & 287 & 0.56 & 88 & 90 & 4.82 & 1.27 & 79 & 1.43 & 0.199 & 1.23 & 413 & 132 & 4,287 & 113 & 121 \\
\hline 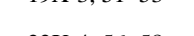 & 401.8 & 54 brown. & 9.52 & 560 & 0.55 & 88 & 93 & 4.77 & 1.26 & 79 & 1.46 & 0.734 & 1.21 & 418 & 142 & 4,411 & 140 & 121 \\
\hline $22 X-4,56-58$ & 432.5 & 55 & 9.21 & 402 & 0.33 & 92 & 227 & 4.25 & 2.20 & 69 & 1.04 & 0.040 & 1.19 & 259 & 142 & 4,507 & 181 & 2038 \\
\hline $22 \mathrm{X}-6,59-61$ & 435.5 & 56 & 9.92 & 344 & 0.38 & 89 & 49 & 5.70 & 1.80 & 69 & 1.35 & 0.038 & 1.1 & 371 & 124 & 4,336 & 139 & 114 \\
\hline $25 \mathrm{H}-1,126-128$ & 459.7 & 57 & 11.59 & 382 & 0.38 & 116 & 205 & 5.25 & 2.63 & 124 & 1.11 & 0.024 & 0.96 & 507 & 144 & 4,476 & 179 & 191 \\
\hline $\begin{array}{l}\text { 156-949C- } \\
4 \mathrm{R}-1,19-21\end{array}$ & 426.0 & 58 & 9.85 & 426 & 0.45 & 99 & 110 & 4.85 & 1.97 & 81 & 1.11 & 0.067 & 1.27 & 326 & 180 & 4,070 & 196 & 169 \\
\hline
\end{tabular}

Table 3. Rare earth element abundance of bulk sediments and authigenic minerals, Sites 948 and 949. ,

\begin{tabular}{|c|c|c|c|c|c|c|c|c|c|c|c|c|c|c|c|c|c|c|}
\hline $\begin{array}{l}\text { Core, section, } \\
\text { interval }(\mathrm{cm})\end{array}$ & $\begin{array}{l}\text { Depth } \\
\text { (mbsf) }\end{array}$ & $\begin{array}{l}\text { Working } \\
\text { no. }\end{array}$ & $\begin{array}{c}\mathrm{Sc} \\
(\mathrm{ppm})\end{array}$ & $\begin{array}{c}\mathrm{Y} \\
(\mathrm{ppm})\end{array}$ & $\begin{array}{c}\mathrm{La} \\
(\mathrm{ppm})\end{array}$ & $\begin{array}{c}\mathrm{Ce} \\
(\mathrm{ppm})\end{array}$ & $\begin{array}{c}\mathrm{Pr} \\
(\mathrm{ppm})\end{array}$ & $\begin{array}{c}\mathrm{Nd} \\
(\mathrm{ppm})\end{array}$ & $\begin{array}{c}\mathrm{Sm} \\
(\mathrm{ppm})\end{array}$ & $\begin{array}{c}\mathrm{Eu} \\
(\mathrm{ppm})\end{array}$ & $\begin{array}{c}\mathrm{Gd} \\
(\mathrm{ppm})\end{array}$ & $\begin{array}{c}\mathrm{Tb} \\
(\mathrm{ppm})\end{array}$ & $\begin{array}{c}\text { Dy } \\
(\mathrm{ppm})\end{array}$ & $\begin{array}{c}\text { Ho } \\
(\mathrm{ppm})\end{array}$ & $\begin{array}{c}\mathrm{Er} \\
(\mathrm{ppm})\end{array}$ & $\begin{array}{c}\mathrm{Tm} \\
(\mathrm{ppm})\end{array}$ & $\begin{array}{c}\mathrm{Yb} \\
(\mathrm{ppm})\end{array}$ & $\begin{array}{c}\mathrm{Lu} \\
(\mathrm{ppm})\end{array}$ \\
\hline \multicolumn{19}{|l|}{$156-948 \mathrm{C}-$} \\
\hline $2 \mathrm{X}-2,85-87$ & 423.2 & 4 & 16.3 & 25.6 & 35.7 & 85.4 & 8.34 & 33.3 & 6.41 & 1.36 & 5.72 & 0.81 & 4.95 & 0.98 & 2.71 & 0.43 & 2.86 & 0.43 \\
\hline $3 \mathrm{X}-3,13-15$ & 436.6 & 5 & 23.9 & 27.2 & 35.9 & 81.9 & 8.96 & 35.9 & 7.01 & 1.73 & 7.02 & 1.01 & 5.85 & 1.18 & 3.13 & 0.45 & 3.02 & 0.46 \\
\hline $4 \mathrm{X}-3,102-106$ & 444.1 & 6 & 21.3 & 21.7 & 30.5 & 67.4 & 7.46 & 30.2 & 5.64 & 1.35 & 5.84 & 0.81 & 4.73 & 0.90 & 2.50 & 0.38 & 2.51 & 0.38 \\
\hline $6 \mathrm{X}-3,127-129$ & 463.7 & 11 & 22.5 & 31.8 & 26.9 & 74.8 & 7.69 & 28.3 & 5.92 & 1.48 & 5.74 & 0.93 & 5.48 & 1.08 & 3.01 & 0.48 & 3.17 & 0.53 \\
\hline $7 \mathrm{X}-3,71-73$ & 472.7 & 12 & 17.1 & 57.3 & 27.3 & 73.5 & 6.72 & 26.3 & 5.31 & 1.43 & 8.03 & 1.02 & 6.72 & 1.62 & 5.10 & 0.93 & 6.80 & 1.08 \\
\hline $9 \mathrm{X}-4,6-9$ & 493.0 & 15 & 44.0 & 4.9 & 2.6 & 6.4 & 0.81 & $\begin{array}{r}2.5 \\
3.6\end{array}$ & 0.86 & $\begin{array}{l}1.43 \\
0.32\end{array}$ & $\begin{array}{l}.0 .03 \\
0.94\end{array}$ & 0.17 & 1.01 & $\begin{array}{l}1.22 \\
0.21\end{array}$ & 0.65 & 0.09 & $\begin{array}{l}0.00 \\
0.75\end{array}$ & 0.11 \\
\hline $9 \mathrm{X}-4,27-30$ & 493.2 & 16 & 28.3 & 27.4 & $\begin{array}{r}24.0 \\
24.3\end{array}$ & $\begin{array}{r}5.4 \\
54.6\end{array}$ & $\begin{array}{l}.01 \\
6.35\end{array}$ & $\begin{array}{r}3.0 \\
25.5\end{array}$ & $\begin{array}{l}0.00 \\
5.49\end{array}$ & 1.45 & 5.72 & 0.91 & $\begin{array}{l}1.01 \\
5.34\end{array}$ & $\begin{array}{l}0.21 \\
1.06\end{array}$ & 3.05 & 0.48 & 3.15 & 0.47 \\
\hline $9 \mathrm{X}-5,53-55$ & $\begin{array}{l}493.2 \\
494.9\end{array}$ & $\begin{array}{l}10 \\
17\end{array}$ & 34.5 & $\begin{array}{l}27.4 \\
11.2\end{array}$ & $\begin{array}{r}24.3 \\
3.6\end{array}$ & $\begin{array}{r}54.0 \\
9.7\end{array}$ & $\begin{array}{l}0.35 \\
1.04\end{array}$ & $\begin{array}{r}2.5 \\
3.9\end{array}$ & $\begin{array}{l}5.49 \\
1.07\end{array}$ & $\begin{array}{l}1.43 \\
0.42\end{array}$ & 1.19 & $\begin{array}{l}0.91 \\
0.22\end{array}$ & $\begin{array}{l}3.34 \\
1.68\end{array}$ & $\begin{array}{l}1.00 \\
0.42\end{array}$ & $\begin{array}{l}3.05 \\
1.40\end{array}$ & $\begin{array}{l}0.48 \\
0.27\end{array}$ & $\begin{array}{l}3.13 \\
2.14\end{array}$ & $\begin{array}{l}0.47 \\
0.35\end{array}$ \\
\hline $\begin{array}{l}9 \times-5,53-55 \\
10 \times-1,74-77\end{array}$ & $\begin{array}{l}494.9 \\
498.8\end{array}$ & 18 & $\begin{array}{l}34.9 \\
30.3\end{array}$ & $\begin{array}{l}11.2 \\
9.6\end{array}$ & $\begin{array}{l}3.6 \\
6.3\end{array}$ & $\begin{array}{r}9.1 \\
15.1\end{array}$ & $\begin{array}{l}1.04 \\
1.87\end{array}$ & $\begin{array}{l}3.9 \\
8.0\end{array}$ & $\begin{array}{l}1.0 / \\
1.85\end{array}$ & $\begin{array}{l}0.42 \\
0.65\end{array}$ & $\begin{array}{l}1.19 \\
2.14\end{array}$ & $\begin{array}{l}0.22 \\
0.32\end{array}$ & $\begin{array}{l}1.68 \\
1.73\end{array}$ & $\begin{array}{l}0.42 \\
0.41\end{array}$ & $\begin{array}{l}1.40 \\
1.19\end{array}$ & 0.27 & $\begin{array}{l}2.14 \\
1.21\end{array}$ & $\begin{array}{l}0.35 \\
0.20\end{array}$ \\
\hline $10 \mathrm{X}-2,44-46$ & 500.0 & 19 ocher & 23.1 & 42.9 & 41.2 & 99.1 & 10.20 & 41.7 & 8.32 & 2.06 & 8.32 & 1.26 & 7.51 & 1.57 & 4.64 & 0.67 & 4.02 & 0.64 \\
\hline $10 \mathrm{X}-2,44-46$ & 500.0 & 19black & 21.6 & 40.6 & 38.3 & 97.9 & 9.48 & 40.4 & 8.35 & 2.13 & 7.94 & 1.18 & $\begin{array}{l}7.09 \\
\end{array}$ & 1.39 & $\begin{array}{l}3.79 \\
\end{array}$ & 0.58 & 3.87 & 0.66 \\
\hline $10 \mathrm{X}-3,110-150$ & 502.4 & 22 & 22.8 & 34.3 & 39.7 & 100.5 & 9.61 & $\begin{array}{l}4.4 \\
43.2\end{array}$ & $\begin{array}{l}. .35 \\
8.38\end{array}$ & $\begin{array}{l}.13 \\
1.94\end{array}$ & $\begin{array}{l}.1 .94 \\
7.78\end{array}$ & $\begin{array}{l}1.10 \\
1.19\end{array}$ & $\begin{array}{l}6.09 \\
6.96\end{array}$ & 1.36 & 3.67 & 0.57 & 3.68 & $\begin{array}{l}0.00 \\
0.58\end{array}$ \\
\hline $11 \mathrm{X}-2,13-15$ & 509.3 & 24black & 19.0 & 25.0 & 38.6 & 105.7 & 9.33 & 36.9 & 6.89 & 1.75 & 5.93 & 0.82 & $\begin{array}{l}0.90 \\
4.77\end{array}$ & $\begin{array}{l}1.00 \\
0.95\end{array}$ & 2.63 & 0.39 & 3.22 & $\begin{array}{l}0.00 \\
0.41\end{array}$ \\
\hline $12 \mathrm{X}-6,3-5$ & 524.9 & 27 greenish & 17.9 & 19.2 & 42.8 & 89.4 & 9.73 & 36.4 & 6.36 & 1.37 & 5.38 & 0.76 & 4.17 & 0.76 & 2.10 & 0.31 & 2.37 & 0.34 \\
\hline $12 X-6,3-5$ & 524.9 & 27 gray & 17.8 & 16.6 & 40.8 & 88.0 & 9.54 & 35.7 & 5.96 & 1.25 & 5.13 & 0.66 & 3.82 & 0.74 & 2.04 & 0.31 & 2.04 & 0.30 \\
\hline $13 \mathrm{X}-\mathrm{CC}, 7-9$ & 536.4 & 28greenish & 19.9 & 20.9 & 45.4 & 103.1 & 10.85 & 41.8 & 7.61 & 1.62 & 6.32 & 0.89 & 5.16 & 0.93 & 2.47 & 0.39 & 2.65 & 0.35 \\
\hline $13 \mathrm{X}-\mathrm{CC}, 7-9$ & 536.4 & 28 gray & 19.1 & 14.1 & 42.2 & 87.4 & 9.27 & 35.3 & 5.86 & 1.10 & 4.55 & 0.61 & 3.33 & 0.62 & 1.82 & 0.26 & 1.96 & 0.30 \\
\hline $15 \mathrm{X}-2,87-89$ & 547.8 & 31 & 19.9 & 27.8 & 56.8 & 1206 & 1238 & 475 & 8.18 & $\begin{array}{l}1.10 \\
1.88\end{array}$ & 7.15 & 0.98 & 6.82 & 1.03 & 2.05 & 0.43 & 285 & 0.39 \\
\hline $17 \mathrm{X}-4,81-84$ & 569.4 & 34 & 19.3 & 30.3 & $\begin{array}{l}39.8 \\
49.4\end{array}$ & $\begin{array}{l}110.0 \\
110.7\end{array}$ & $\begin{array}{l}12.90 \\
11.91\end{array}$ & 47.3 & $\begin{array}{l}0.10 \\
8.48\end{array}$ & $\begin{array}{l}1.80 \\
1.97\end{array}$ & 8.04 & $\begin{array}{l}0.90 \\
1.11\end{array}$ & $\begin{array}{l}0.22 \\
6.62\end{array}$ & 1.25 & 3.38 & 0.45 & 3.20 & 0.45 \\
\hline $19 X-6,72-75$ & 591.0 & 36 & 16.1 & 27.0 & 52.7 & 113.2 & 11.39 & 45.2 & 8.06 & 1.80 & 6.73 & 1.00 & 5.60 & 1.03 & 2.74 & 0.41 & 2.69 & 0.46 \\
\hline \multicolumn{19}{|l|}{ 156-949B- } \\
\hline 4X-CC, $19-21$ & 275.0 & 42 & 24.8 & 14.8 & 15.7 & 37.8 & 4.32 & 17.2 & 3.48 & 0.87 & 3.36 & 0.53 & 3.35 & 0.66 & 1.81 & 0.25 & 1.91 & 0.28 \\
\hline $5 \mathrm{X}-2,8-10$ & 284.4 & 43matrics & 33.1 & 20.1 & 17.9 & 44.0 & 5.13 & 21.8 & 4.91 & 1.2 & 4.8 & 0.78 & 4.59 & 0.87 & 2.27 & 0.36 & 2.30 & 0.37 \\
\hline $5 \mathrm{X}-2,8-10$ & 284.4 & 43greenish & 25.0 & 15.9 & 9.3 & 26 & 3.07 & 14.6 & 3.41 & 0.9 & 3.8 & 0.60 & 3.41 & 0.64 & 1.74 & 0.27 & 1.96 & 0.27 \\
\hline $5 X-5,3-5$ & 288.8 & 44 & 22.0 & 26.5 & 28.4 & 72.8 & 7.94 & 32.1 & 6.10 & 1.64 & 6.24 & 0.97 & 5.71 & 1.06 & 3.03 & 0.45 & 2.92 & 0.48 \\
\hline $5 X-7,40-42$ & 292.2 & 45 & 24.9 & 33.1 & 28.3 & 68.2 & 7.78 & 31.6 & 6.58 & 1.74 & 7.04 & 1.10 & 6.53 & 1.31 & 3.58 & 0.57 & 3.74 & 0.56 \\
\hline
\end{tabular}



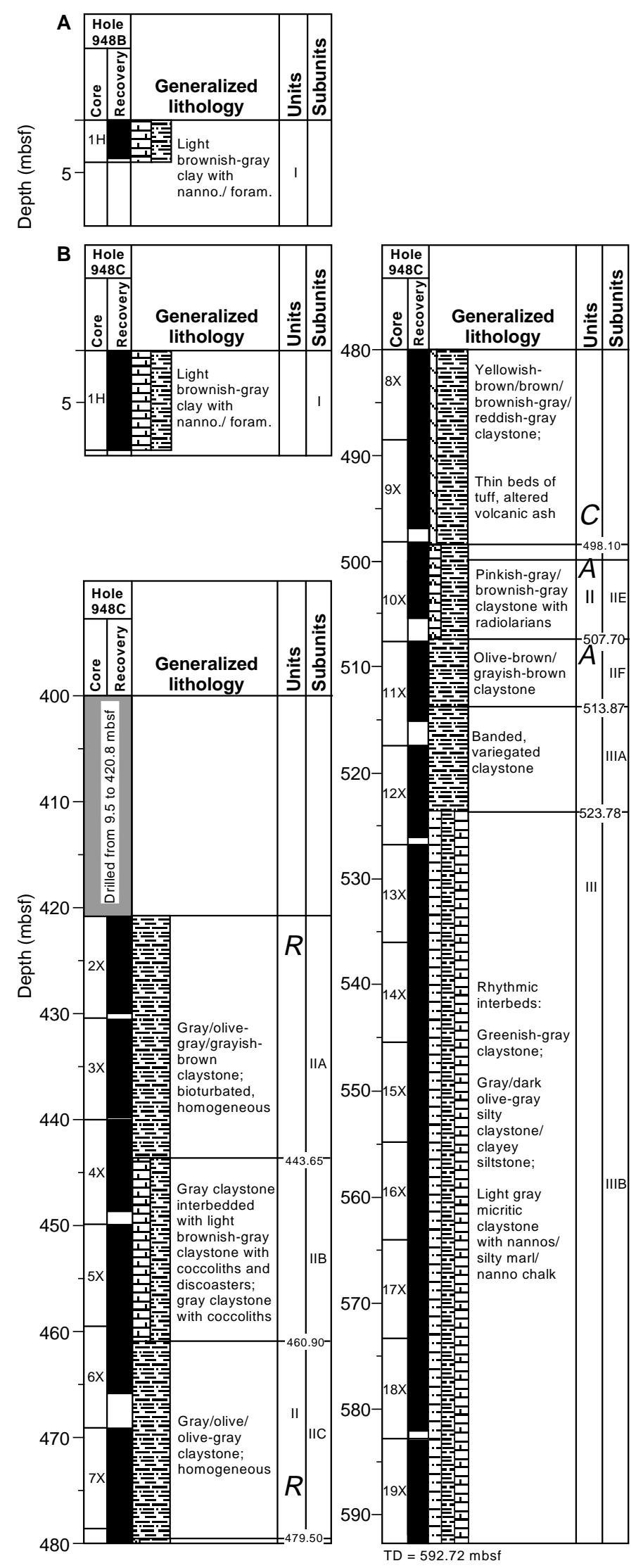

Figure 3. Lithostratigraphic summary for Site 948, adapted from Shipboard Scientific Party (1995b). A. Hole 948B. B. Hole 948C. In "Units" column, "R," "A," and "C" show occurrences of rhodochrosite, amorphous $\mathrm{Mn}$ oxide, and $\mathrm{Cu}$ enrichment. 

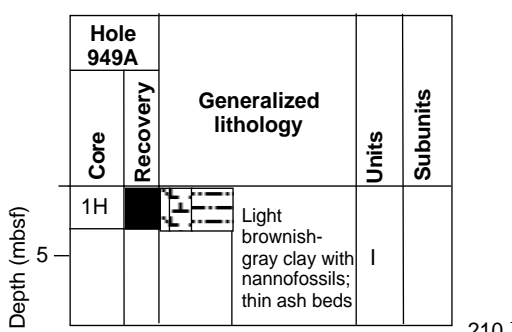

\section{Hole
$949 \mathrm{~B}$}

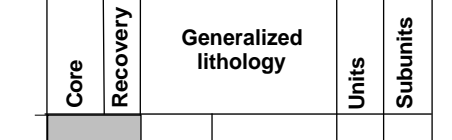

210

220

230

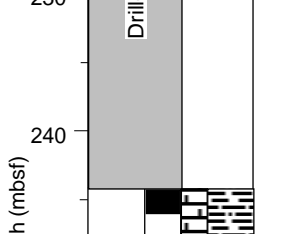

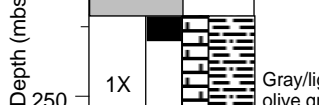

260

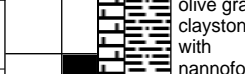

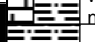
0

280

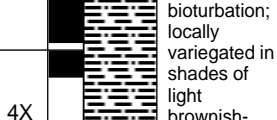

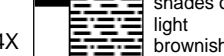

290
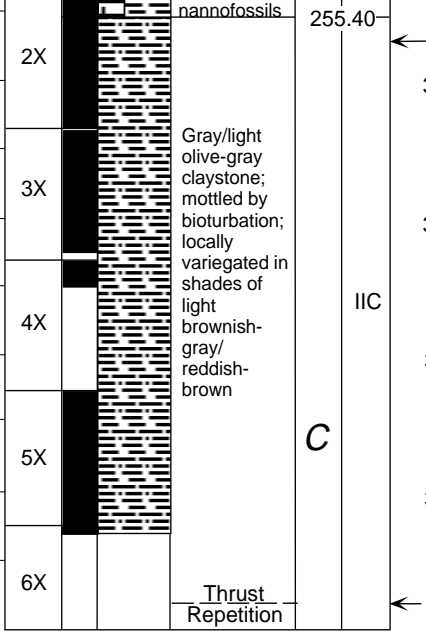

\section{Hole}

Hole
$949 \mathrm{~B}$
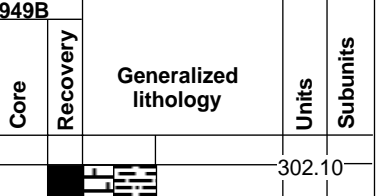

$310-$
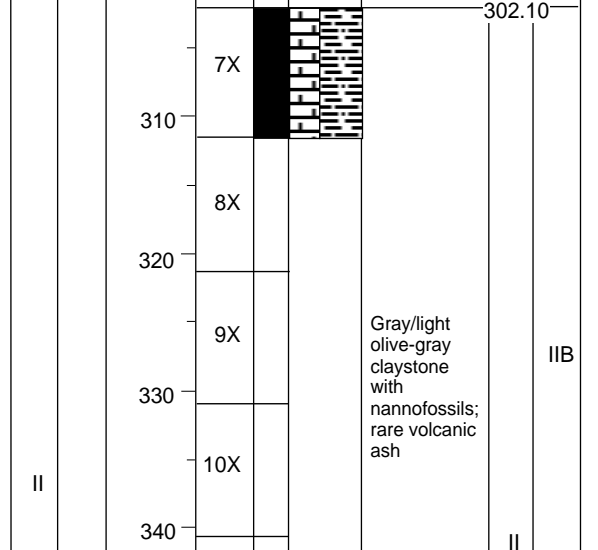

IIB
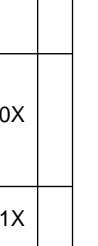

\begin{tabular}{|l|l|}
\hline $11 X$ & \\
\hline $12 X$ &
\end{tabular}

with
nannofossils:

rare volcanic

$13 x$

$\leftarrow$

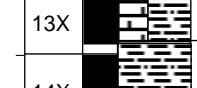

둑

둘훌

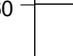

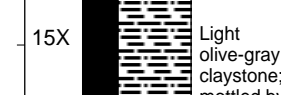

$370-1 \quad \begin{aligned} & \text { claystone; } \\ & \text { mottled by } \\ & \text { bioturbation; }\end{aligned}$

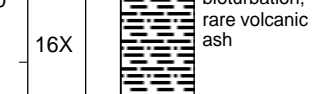

IIC

380

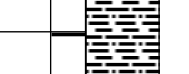

390
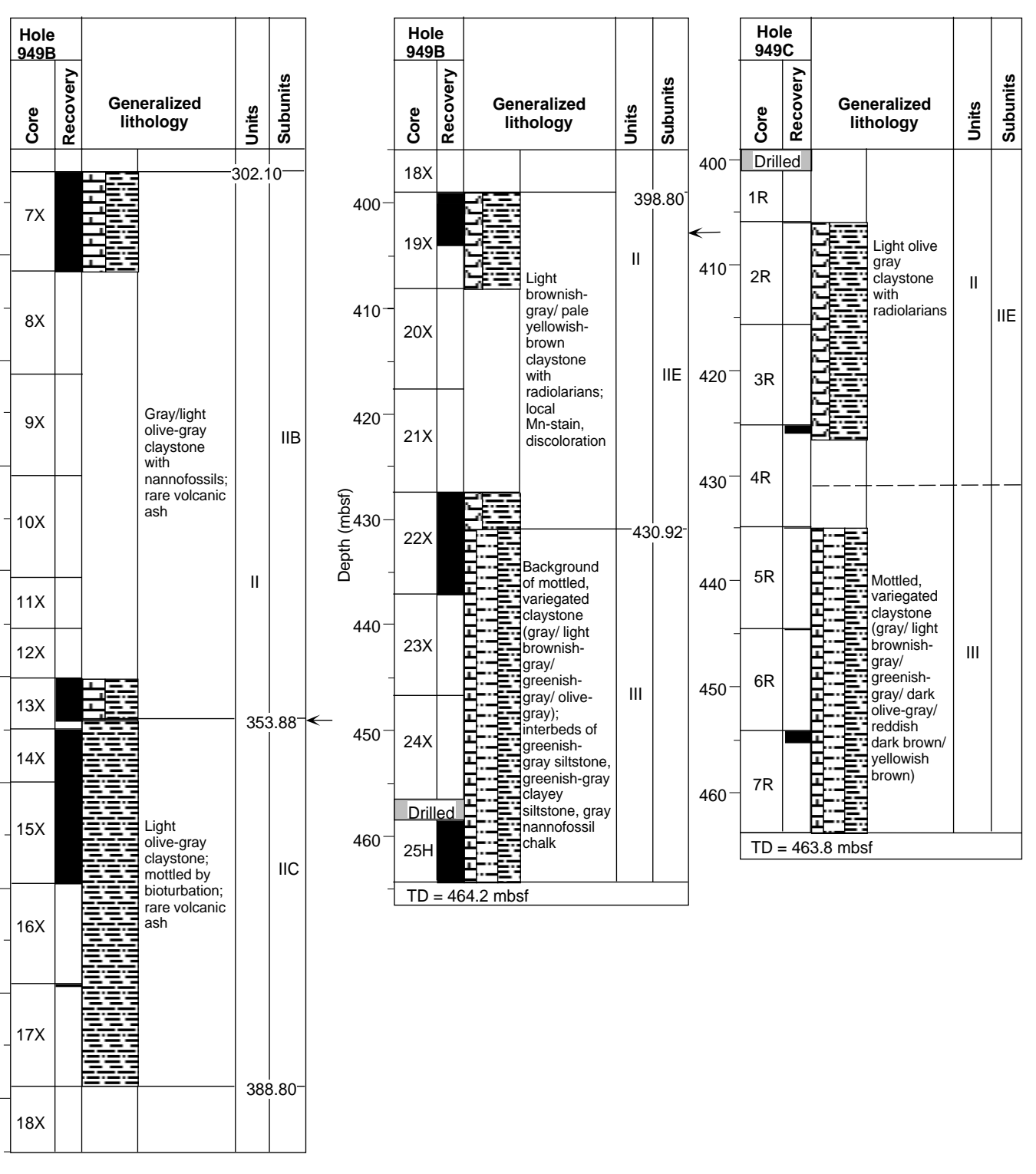

$400-$

$19 x$

$1 \mathrm{R}$

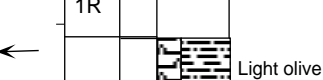

$10-$

$0-$

Light
brownish-
gray/ pale
yellowish-
chasson

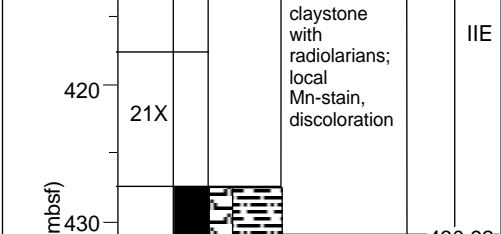

$420-3 R$

with

$430^{-}-4 \mathrm{R}$

$4 \mathrm{R}$

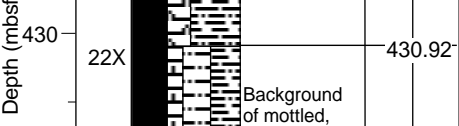

440- 5R

440

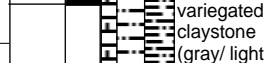

- in: claystone

$450-6 \mathrm{R}$,

450

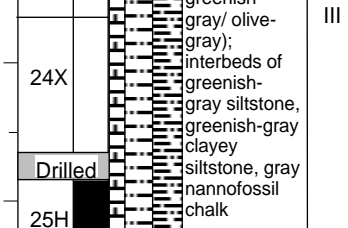

$460-$

$460-$

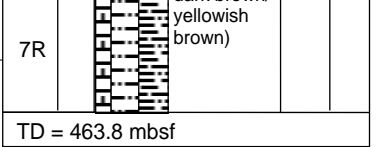

$\mathrm{TD}=464.2 \mathrm{mbsf}$ 

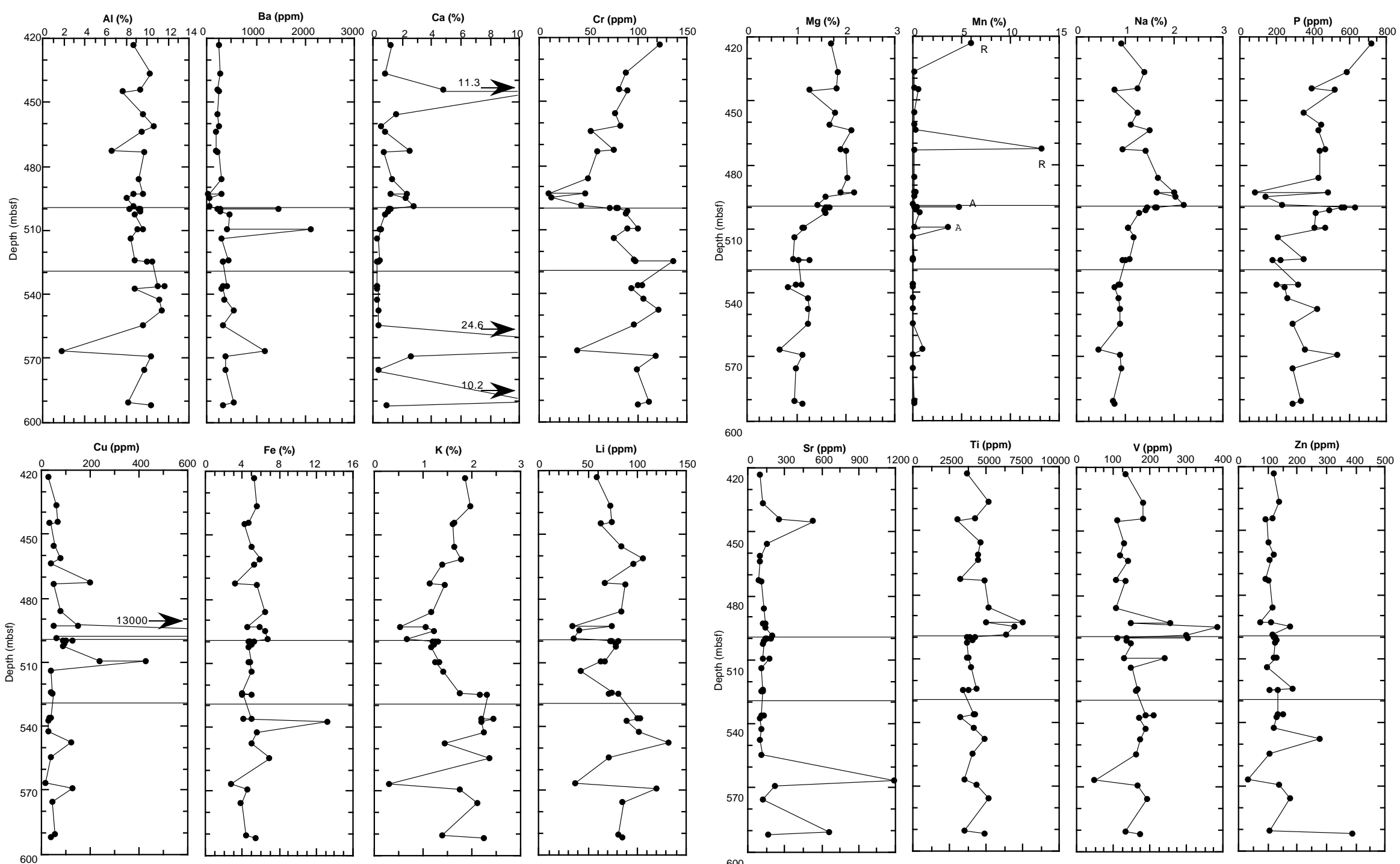

$\mathrm{Ti}$ (ppm)

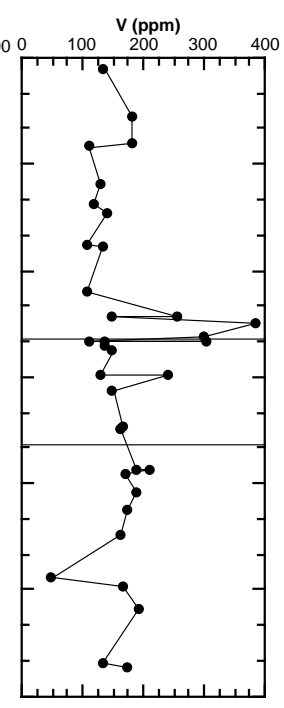

$\mathrm{Zn}(\mathrm{ppm})$

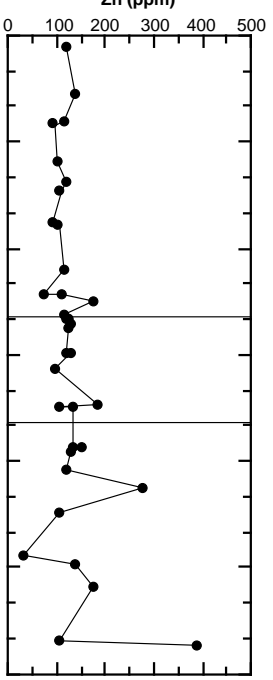



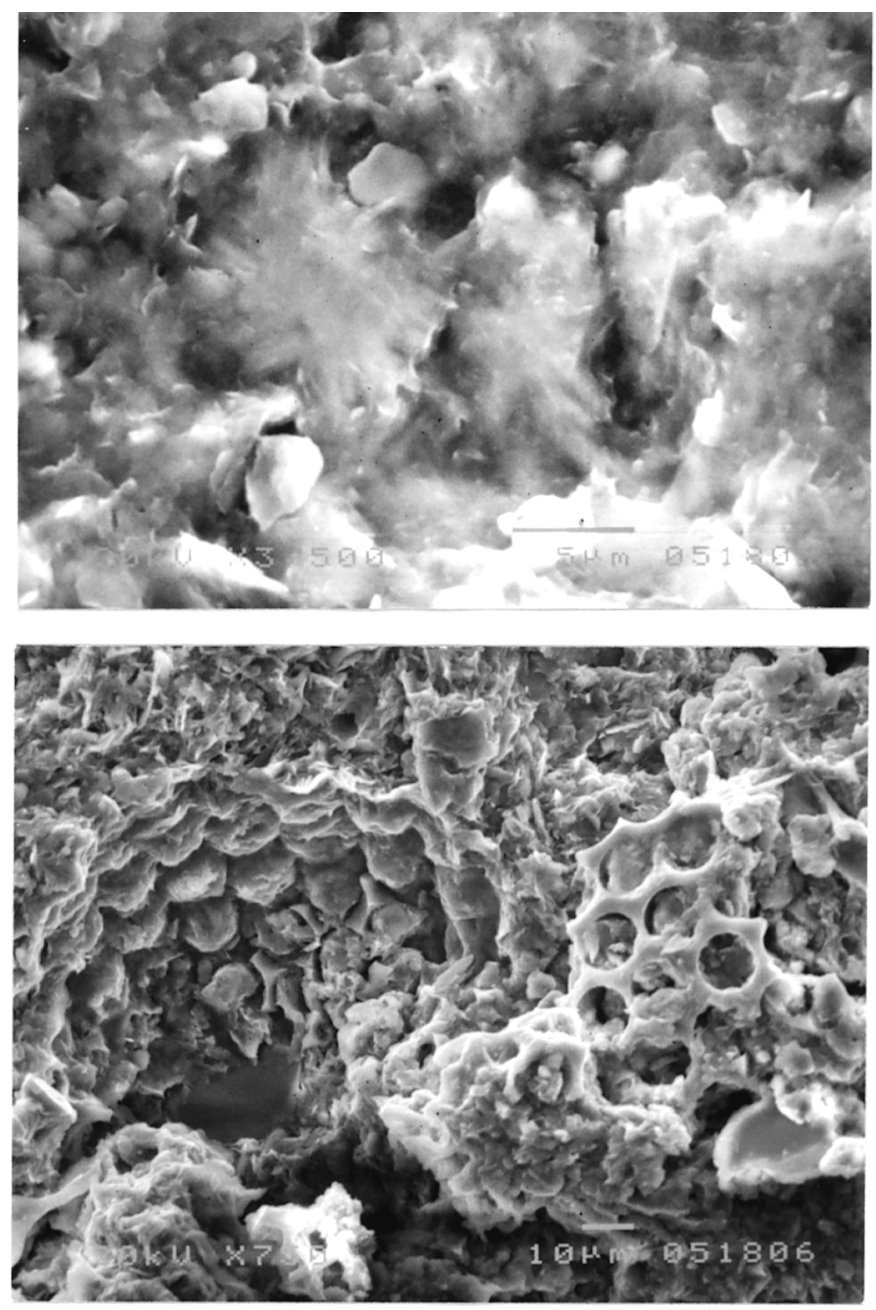

Figure 6. Scanning electron photomicrographs of various morphologies of black amorphous Mn minerals (Sample 156-948C-10X-2, 44-46 cm). Upper part: typical texture of amorphous black Mn oxides. Lower part: Mn oxides forming molds of siliceous fossil tests.

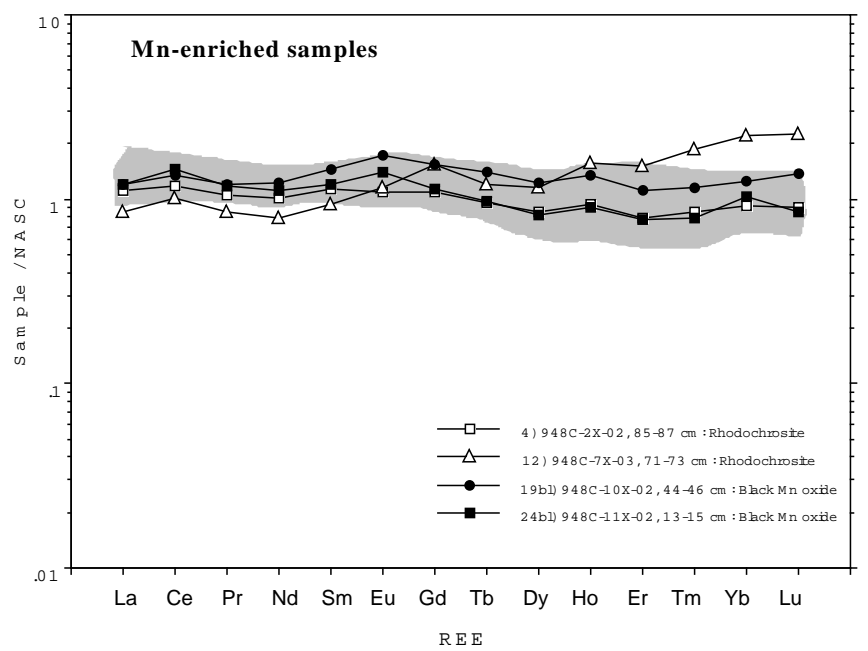

Figure 7. REE abundance of Mn-enriched samples in Leg 156 (open symbols = rhodochrosite-bearing, solid symbols $=$ amorphous Mn oxide-bearing parts). The shaded portion shows the range of REE abundances of typical sediments from Leg 156. REE abundances of North American Shale Composites (NASC) were adapted from Haskin et al. (1968) and Gromet et al. (1984).

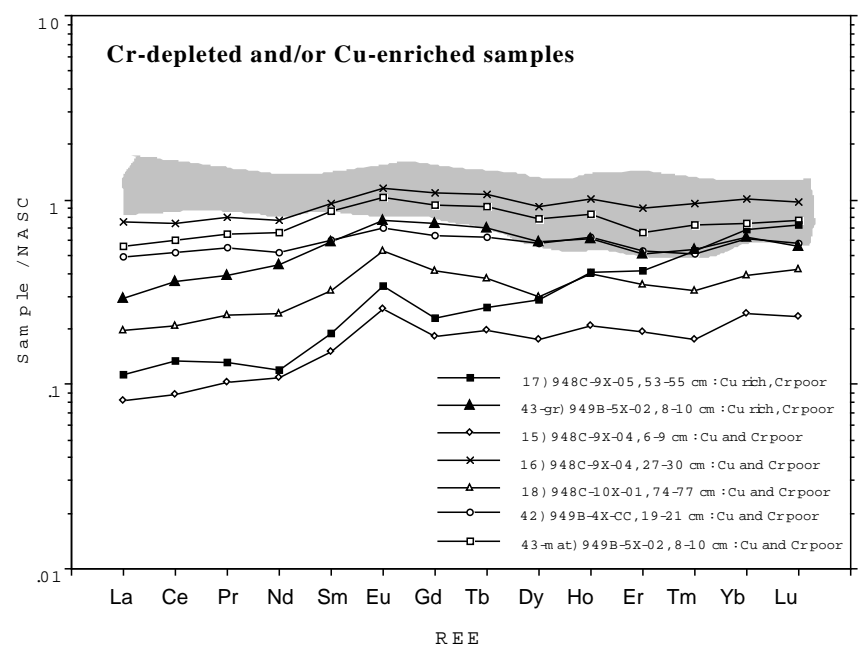

Figure 8. REE abundances of $\mathrm{Cr}$-depleted and/or $\mathrm{Cu}$-enriched samples from Leg 156. All the samples plotted here have low $\mathrm{Cr}$ contents, $<50 \mathrm{ppm}$. Solid symbols are $\mathrm{Cr}$-depleted and $\mathrm{Cu}$-enriched samples. Open symbols show $\mathrm{Cr}$ and $\mathrm{Cu}$-depleted samples. The shaded portion represents the range of REE abundance of typical sediments from Leg 156 . 


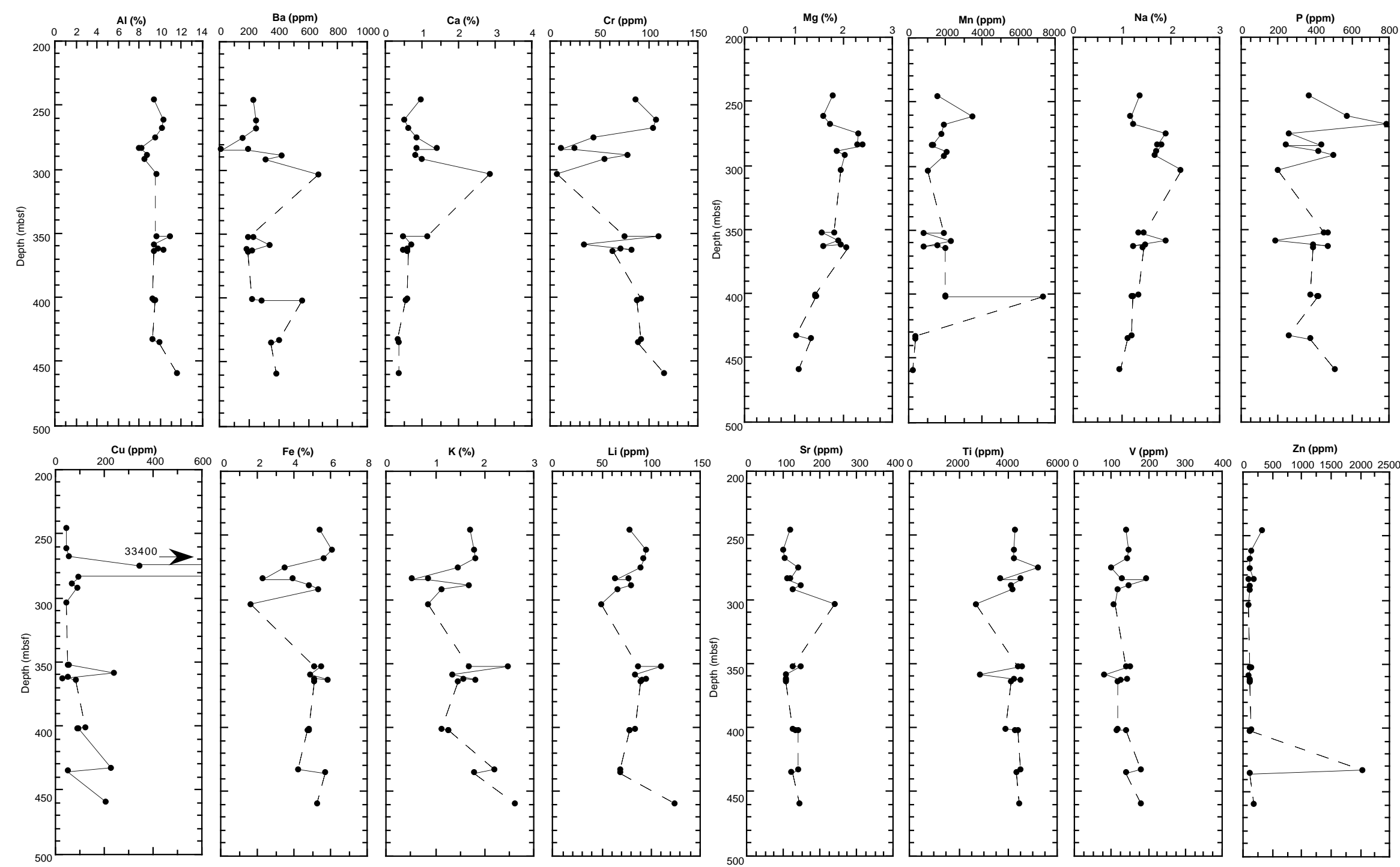

Figure 9. Element compositions of bulk sediments and authigenic minerals from Site 949. Depth shown by dashed line indicates range of no recovery. 

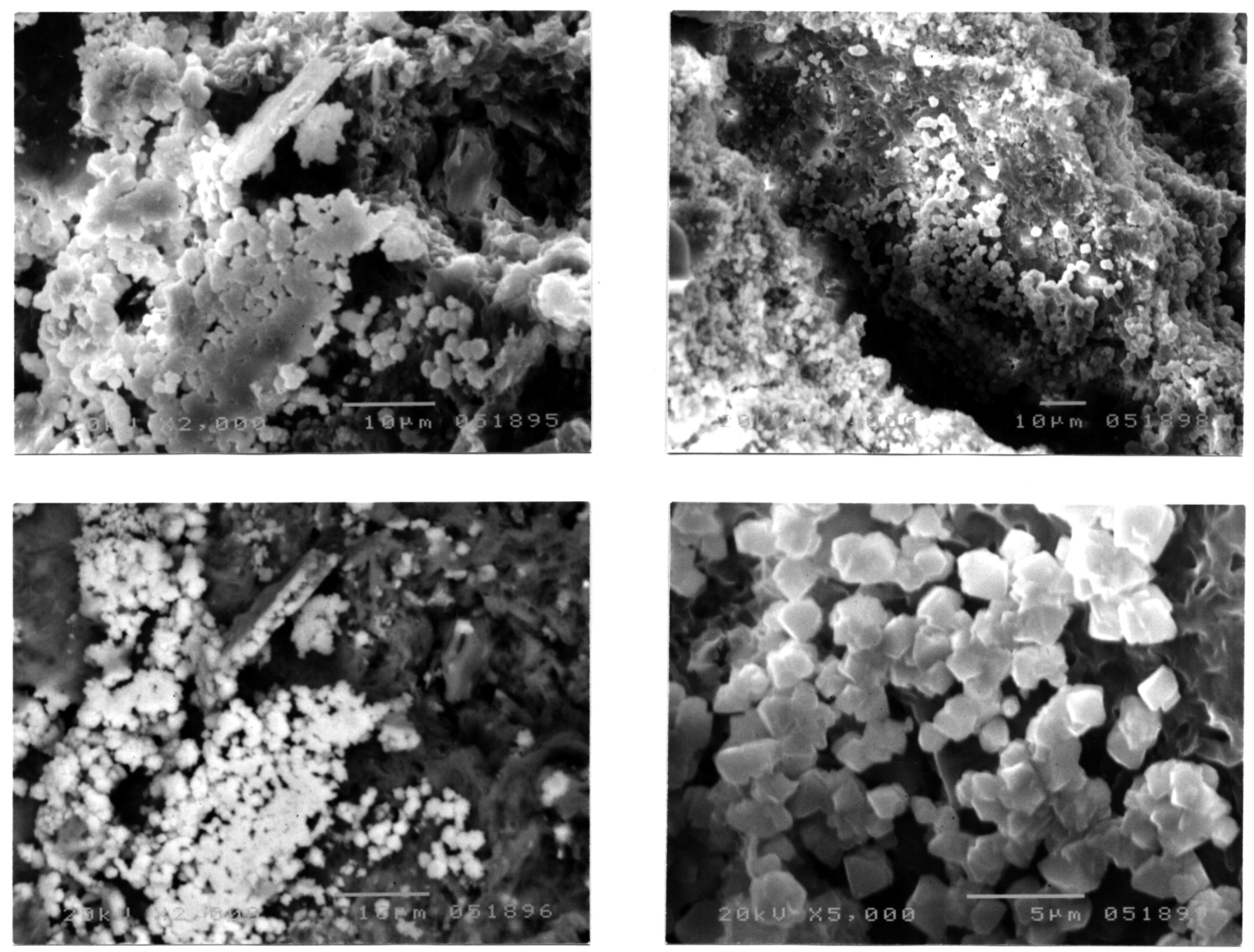

Figure 10. Scanning electron photomicrographs and component image of various morphologies of Cu minerals (Sample 156-949B-5X-2, 8-10 cm). Upper parts: aggregate of single grains. Lower left: component image of same area as the upper left photomicrograph. White parts correspond to Cu minerals. Lower right: close-up of upper right photomicrograph. 